\title{
III. On the theoretical determination of the motion of fluids
}

\section{Rev. J. Challis}

To cite this article: Rev. J. Challis (1831) III. On the theoretical determination of the motion of fluids, Philosophical Magazine Series 2, 9:49, 7-11, DOI: 10.1080/14786443108647521

To link to this article: http://dx.doi.org/10.1080/14786443108647521

曲 Published online: 01 Jun 2009.

Submit your article to this journal \lceil

Џll Article views: 3

Q View related articles $\sqsubset$ 
on this branch at all prepared for publication. A paper of his, published in the Transactions of the Geological Society, contains very valuable contributions towards the history of our fossil Belemnites, and has been most favourably referred to by the French author who has subsequently published the standard monography of that department.

Mr. Miller's talents have been highly estimated by the ablest of our naturalists and geological writers. Professor Blumenbach, Baron Cuvier, MM. Latreille and D'Aubigné, have expressed in letters to him high commendation of his works. Professor Buckland obtained his assistance in arranging the valuable collection of organic remains belonging to the Ashmolean Museum at Oxford. The same Professor, in his very interesting paper "on the recent discovery in this country of fossil remains belonging to the flying reptile the Pterodactylus, mentions that Mr. Miller first suggested to him the possibility, thus confirmed, that the fossil bones commonly supposed to belong to birds really appertained to that animal. And Mr. Conybeare, while drawing up the lists of the organic remains in our strata, which are given in his "Outlines," was in the common habit of appealing to Mr. Miller's authority.

In surveying the results of $\mathrm{Mr}$. Miller's scientific acquirements and of his exertions, we must not forget the important benefits rendered by him to the Museum of the Institution of which he was Curator. It may safely be affirmed, that the history of similar collections does not present another instance in which so rapid a progress has been made in accumulating the varied stores connected with such undertakings; and the rapidity of this progress must undoubtedly be ascribed in a great measure to the energy and zeal of the Curator in the service, and to the interest which he so well knew how to communicate to those with whom he came into intercourse.

III. On the theoretical Determination of the Motion of Fluids. By the Rev. J. Chaldis, Fellow of Trinity College, Cambridge, and of the Camb. Phil. Soc.*

SUPPOSE $x, y, z$, to be the coordinates of any particle of $N$ a fluid mass in motion, at a given time $t$, and $u, v$, $w$, the velocities of the particle in the directions of the axes of $x, y, z$, respectively, at that time. The general investigation of the motion of fluids conducts to a case of very extensive application, in which $u d x+v d y+r d z$ is a complete differential of a function of $x, y, z$, which may also contain $t$. In a commu-

* Communicated by the Author. 
nication to the Phil. Mag. and Annals of Philosophy for August 1829, which contained several inaccuracies, I made an assertion respecting this case of fluid motion, the correctness of which subsequent consideration has only tended to confirm; viz. that when $u d x+v d y+v d z$ is an exact differential, the whole motion is such that the motion of each elementary portion of the fluid is directed to a fixed or moveable centre. The course of the reasoning by which this proposition may be established is I conceive such as follows. We know from the theory of partial differential equations, that their integrals, whether we can obtain them exactly or not, must contain arbitrary functions. The arbitrariness of which we are informed by pure analysis, has a signification in the applications of the functions to physical questions. Thus the existence of arbitrary functions in the integrals of the equations which determine the motion of fluids, is the proper proof that we can give to the fluid any motion we please; and this is an evident consequence of one of the fundamental principles in the investigation of the motion, - the perfect mobility of the particles. The forms of the functions depend on the particular motion we choose to give to the fluid by vessels, pipes, or other means. But however irregular we may cause the motion to be, it may still be conceived to be composed of elementary motions, which obey the law of continuity, independently of our will, just as a line, however broken and irregular, may be conceived to be made up of elementary portions which are straight lines. Absolute discontinuity is inconceivable. The law of these motions will be independent of time and position, and dependent only on the nature of the fluid. Hence, to learn whether the motion be really so composed, it will be necessary, after having obtained the complete integral of the equation expressing the continuity of the fluid, to ascertain whether the arbitrary functions which the integral contains, can be shown to have a particular form, when discussed on the supposition that the origins of the time and coordinates are not fixed. This will in general require the solution of a functional equation. An instance of this reasoning was given in the communication above mentioned, for the case in which the motion is in space of two dimensions. From the complete integral of $\frac{d^{2} \phi}{d x^{2}}+\frac{d^{2} \phi}{d y^{2}}$ $=0$, a particular form of the arbitrary functions was obtained, which indicated that the velocity was directed to a centre and varied inversely as the distance from the centre. I have since found that the complete integral of $\frac{d^{2} \phi}{d x^{2}}+\frac{d^{2} \phi}{d y^{3}}+\frac{d^{2} \phi}{d z^{2}}=0$, which M. Poisson has expressed by definite integrals (Mém. de l'Acad. 
l'Acad. des Scien. Ann. 1818), also conducts to a particular form of the arbitrary functions, when treated according to the same principles : and from the discussion it appears that $\phi$ has the same value as would be obtained by integrating $\frac{d^{2} \varphi}{d x^{2}}+$ $\frac{d^{2} \phi}{d y^{2}}+\frac{d^{3} \phi}{d z^{2}}=0$, on the supposition that $\varphi$ is a function of $\sqrt{x^{2}+y^{2}+z^{2}}$ or $r$, and $t$; in which case the velocity is directed to or from a centre, and varies inversely as the square of the distance from the centre. The instances in which $u d x+v d y+w d z$, or $d \Phi$, is known not to be a complete differential of a function of $x, y, z$, confirm the view here taken. When a mass of incompressible fluid revolves uniformly, without changing form, about a fixed axis, $d \phi$ is not an exact differential; and it is plain that the motions of elementary portions of the fluid are not directed to fixed or moveable centres, because the whole mass moves in such a manner that it may be considered solid. Again, Euler has shown that $d \phi$ is not an exact differential, when a mass of incompressible fluid revolves round a fixed axis, and the velocity is any function of the distance from the axis, and yet that the general equations are satisfied by this case of motion. We may in this instance conceive the fluid to be divided into portions, included between cylindrical surfaces indefinitely near each other, having in common the axis of revolution: the motion of each portion will be the same as if it were solid. No part of the motion will be such as is directed to a fixed or moveable centre, that is, the motion will not be that which peculiarly belongs to fluids.

The principle I have endeavoured to establish, will only in particular cases facilitate the solution of hydrodynamical problems, on account of the difficulty of ascertaining the centres to or from which the motion is directed. The following example, selected for illustration, may perhaps be interesting; because no principles, that I am aware of, have hitherto been advanced by which such a problem could be solved.

Water contained in a conical vessel, the axis of which is vertical, descends with its upper surface always horizontal: It is required to find the velocity and direction of the velocity at any given point in the interior of the fluid mass at a given time, when the velocity of the descending surface is given.

In this example, all the particles must be moving in vertical planes passing through the axis of the cone: hence the centres to which the motion is directed, must be situated in the axis. The integral of $\frac{d^{2} \phi}{d x^{2}}+\frac{d^{2} \phi}{d y^{2}}+\frac{d^{2} \phi}{d z^{2}}=0$, supposing $\phi$ to be
N.S. Vol. 9. No. 49. Jan. 1831.
C
a function 
a function of $r$ and $t$, is $\varphi=\frac{\mathrm{F}(t)}{r}+f(t)$, and the velocity $\omega=\frac{d \varphi}{d r}=-\frac{F(t)}{r^{2}}$. From what has been said, these expressions for $\phi$ and $\omega$ will apply to any point of a mass of fluid, moving so that $d \phi$ is an exact differential. But in general $\mathrm{F}(t)$ and $f(t)$ can be considered constant at a given time only for values of $r$ restricted within limits indefinitely near each other. Let $r^{\prime}$ be a value indefinitely near to $r$. Then $\phi^{\prime}-\phi=\mathrm{F}(t)\left(\frac{1}{r^{\prime}}-\frac{1}{r}\right)=-\frac{\mathrm{F}(t)}{r^{2}} \cdot\left(r^{\prime}-r\right)=\omega\left(r^{\prime}-r\right)$.

Here $r^{\prime}-r$ may be considered the increment $d s$ of a line $s$, drawn continually in the direction of the motions of the particles through which it passes. Hence $d \phi=\omega d s$; and $\phi=f \omega d s+\chi(t)$, the integral being taken in regard to an arbitrary portion of the line $s$. The two expressions for $\phi$ thus obtained, have a relation to each other, analogous to that between the two expressions which the general and the particular solutions of a differential equation of the first order give for the same variable. By equating these values of $\phi$,

$$
\begin{gathered}
\frac{\mathrm{F}(t)}{r}+f(t)=f \omega d s+\chi(t) \\
\therefore \omega=-\frac{\mathrm{F}(t)}{r^{2}}=-\frac{(f) \omega d s}{r}-\frac{\chi(t)-f(t)}{r} .
\end{gathered}
$$

But in the example before us, if $y=$ the distance of any point from the axis, $r$, being the length of the portion of a tangent to $s$ intercepted between the point and the axis, will be $y \frac{d s}{d y}$. Therefore,

$$
\begin{aligned}
\omega & =-\frac{d y}{y d s}(f \omega d s+\chi(t)-f(t)) \\
\text { or, } \quad-\frac{d y}{y} & =\frac{\omega d s}{f \omega d s+\chi(t)-f(t)}
\end{aligned}
$$

Hence $\frac{c}{y}=f \omega d s+\chi(t)-f(t)$; and $\therefore \omega=-\frac{c d y}{y^{2} d s}$.

Now the particles in contact with the surface of the cone must move in straight lines directed to its vertex : and if $2 \alpha$ $=$ its vertical angle, $\frac{d y}{d s}=\sin \alpha$. Hence $\omega=-\frac{c \sin \alpha}{y^{2}}$ that is, the velocity varies inversely as the square of $y$, and consequently inversely as the square of the distance from the vertex. Therefore if we conceive a conical surface to have the same vertex and axis as that which contains the fluid, and to have a vertical angle, less by an indefinitely small angle than $2 \alpha$, the fluid contiguous to the containing surface will move as if included 
included between the two surfaces. Similar reasoning may be applied to the fluid contiguous to this new containing surface, and so on throughout the whole of the mass. From this we infer that the motion is at every point directed to the vertex of the given cone. Also, let $\mathrm{V}=$ the velocity of the vertical descent of the horizontal surface, and $h$ its distance from the vertex of the cone, and let us consider a point on this surface, at which the direction of the velocity makes an angle $\theta$ with the axis. The velocity at this point $=\mathrm{V} \sec \theta$ and $\mathrm{V} \sec \theta$ $=-\frac{c}{y^{8}} \cdot \frac{d y}{d s}=-\frac{c \sin \theta}{h^{2} \tan ^{2} \theta^{\circ}}$. Hence $-c=\frac{h^{2} \mathrm{~V} \sin \theta}{\cos ^{3} \theta}$. Therefore in general $\omega=\frac{\mathrm{V} \sin ^{2} \theta}{\cos ^{3} \theta} \cdot \frac{h^{2}}{y^{2}}$. If $\xi=$ the distance of any point from the vertex $\rho \sin \theta=y$, and

$$
\omega=\frac{\mathrm{v} h^{2}}{\cos \theta} \cdot \frac{1}{\rho^{2} \cos ^{2} \theta} .
$$

As the vertical velocity $\omega \cos \theta$ varies as $\frac{1}{(\rho \cos \theta)^{2}}$, it is the same at all points of a plane perpendicular to the axis. Hence the fluid descends in parallel slices; that is, a portion which at any instant is included between horizontal planes will always be included between horizontal planes.

Trin. Coll. Camb. Nov. 13, 1830.

IV. Remarks on the Geology of the Banks of the Tweed, from Carham, in Northumberland, to the Sea Coast at Berwick. By N. J. Wrinch, Esq. Secretary of the Natural History Society of Nerwcastle-upon-Tyne*.

THE rocky strata which border the Tweed from Carham Bourn, where the river begins to form the boundary between Northumberland and Scotland, to the sea shore at Berwick, appearing to be associated in a manner so different from the order generally considered by geologists as the natural arrangement, will oblige me to abstain from theory altogether in the following remarks. It is, therefore, my intention to lay before the Society merely a series of notes lately made during an examination of the north-eastern termination of our district, accompanied by specimens which will serve to assist in verifying the correctness of the observations. A superficial view of the banks of this beautiful river presents a succession of eminences, I can scarcely call them hills, chiefly composed of diluvium, con-

* Read before the Natural History Society of Newcastle-upon-Tyne, on the 20th of July last; and now reprinted from the Transactions of that Society.

C 2

taining 